\title{
Fabricación de matrices de corte con aceros rápidos para herramientas mediante metalurgia de polvos ${ }^{(\bullet)}$
}

\author{
S. Talacchia ${ }^{(*)}$, J. Amador ${ }^{(*)}$ y J. Urcola ${ }^{(* *)}$ \\ Resumen Se estudia la fabricación de matrices de corte de aceros rápidos para herramientas mediante procesos \\ de metalurgia de polvos. Los polvos de los aceros T42 con contenidos de carbono y vanadio modifi- \\ cados, M3/2 y M2, obtenidos por atomización en agua, se compactaron de tal modo que quedaron \\ dotados de formas geométricas complejas. Después de sinterizados en una atmósfera industrial 90 \\ $\mathrm{N}_{2}-9 \mathrm{H}_{2}-1 \mathrm{CH}_{4}$, se trataron térmicamente en medios de distintas severidades. Tras el temple desde \\ temperaturas de austenización cercanas a la temperatura óptima de sinterizado, se lograron durezas \\ de hasta $1.100 \mathrm{HV}$. Se determinaron, también, las contracciones sufridas por las piezas sinterizadas.
}

Palabras clave: Metalurgia de polvos. Acero rápido para herramientas. Matriz de corte. Sinterización. Tratamientos térmicos.

\section{High speed tool steel cut off dies made using powder metallurgy techniques}

\begin{abstract}
The present work summarises the results obtained by compacting water atomised powders of T42 modified carbon and vanadium, M3/2 and M2 high speed steels. The powders were compacted under complex forms (cut off dies) and sintered under a $90 \mathrm{~N}_{2}-9 \mathrm{H}_{2}-1 \mathrm{CH}_{4}$ industrial atmosphere. Heat treatments in different severity media were performed. Hardness of $1,100 \mathrm{HV}$ can be obtained in the case of T42 modified steel. Also, the shrinking of the sintered specimens was study.
\end{abstract}

Keywords: Powder metallurgy. High speed steel. Cutting die. Sintering. Heat treatments.

\section{INTRODUCCIÓN}

Los materiales utilizados en la fabricación de matrices de estampación son, mayoritariamente, aceros que deben reunir ciertas características especiales, tales como alta resistencia mecánica, dureza, tenacidad, resistencia al desgaste, ductilidad, templabilidad, etc. Se utilizan aceros con elevados porcentajes de carbono, aleados con diferentes elementos, como, por ejemplo, aceros al $\mathrm{Cr}$, al $\mathrm{Cr}-\mathrm{Mo}$, al $\mathrm{W}-\mathrm{Cr}$, al V, todos ellos con una gran capacidad de endurecimiento, lo que hace posible la obtención de las propiedades antes mencionadas.

El elevado porcentaje de carbono, sumado a la presencia de elementos formadores de carburos y/o

\footnotetext{
(•) Trabajo recibido el día $1^{\circ}$ de junio de 1995.

(*) Escuela Superior de Ingenieros. Universidad de Navarra. $P^{\circ}$ Manuel de Lardizábal, s/n 20009 San Sebastián. (España).

(**) Centro de Investigaciones Técnicas de Guipúzcoa. (C.E.I.T.). $\mathrm{P}^{\mathrm{o}}$ Manuel de Lardizábal, 15. Apartado 1555. 20009-San Sebastián (España).
}

nitruros, traerá como consecuencia, ya sea durante la colada o durante los tratamientos de endurecimiento, la formación de carburos primarios y secundarios, respectivamente, que pueden clasificarse, de forma general, en carburos ricos en $\mathrm{W}$, Mo y Fe $\left(M_{6} C\right)$, carburos ricos en Mo $\left(M_{2} C\right)$, carburos ricos en $\mathrm{Cr}\left(\mathrm{M}_{\mathrm{X}} \mathrm{C}_{\mathrm{Y}}\right)$ y carburos ricos en $\mathrm{V}(\mathrm{MC})$. Cada uno de los cuales confiere al material distintas propiedades.

El proceso comercial más utilizado para la obtención de estas piezas es el mecanizado de barras obtenidas a partir de solidificación más deformación en caliente, lo que implica costos derivados del alto porcentaje de pérdida de material, de la utilización de mano de obra y de la necesidad del uso de maquinaria especial (para torneado, roscado, fresado, taladrado, etc).

En los aceros obtenidos por colada convencional, como resultado de una segregación pronunciada las microestructuras resultan heterogéneas, con carburos de gran tamaño, lo que ocasiona anisotropía de las propiedades mecánicas, problemas de 
control del tamaño de grano y variaciones de dureza en una misma pieza al someterla a distintos tratamientos térmicos, etc.

En el caso de la fabricación de herramientas por medio de la metalurgia de polvos (PM), éstas se pueden obtener mediante distintos procesos, entre los cuales cabe mencionar el atomizado por agua o por gas. La rápida solidificación de las partículas atomizadas elimina los problemas de segregación, con lo cual pueden retenerse en solución mayores porcentajes de elementos de aleación que los permitidos en una colada convencional. Además, se obtienen microestructuras muy finas con distribución y tamaño de carburos uniformes. Como consecuencia de esto, es posible controlar distintos parámetros, tales como dureza homogénea en toda la pieza, control dimensional durante los tratamientos térmicos, menores tamaños de grano, propiedades mecánicas isótropas, etc.

Dentro de las diferencias existentes entre polvos atomizados por agua o por gas, se puede mencionar que, con los primeros, se obtienen partículas de forma irregular que pueden compactarse en frío con formas variadas. Los compactos así obtenidos tienen alta resistencia mecánica y la capacidad de soportar su forma por sí mismos. Inmediatamente después de la compactación, pueden sinterizarse.

En los polvos atomizados por gas, las partículas son esféricas, por lo que, como paso previo a su compactación en caliente (por HIP, extrusión, laminación o forjado), deben encapsularse.

Una vez compactados, es posible encontrar variaciones de densidad a lo largo del compacto, debidas fundamentalmente a dos causas (1 y 2).

Una de estas causas es que, como consecuencia de la compactación uniaxial, disminuye la densidad del compacto a medida que se avanza hacia el interior del mismo, por lo que, para formas muy esbeltas (relación altura/diámetro $>5$ ), se puede apreciar una variación de la sección alrededor de la zona central. De ahí la utilización de un doble actuador en la prensa que evite grandes diferencias entre los dos extremos de la pieza.

La segunda causa son la variaciones de densidad debidas a formas complejas de la matriz de compactación, como puede ser la existencia de zonas curvas o variaciones bruscas de sección. Éstas se resuelven, en cada caso particular, mediante un estudio de las formas concretas, pero habitualmente obligan a la división de los punzones en varias partes.

En la práctica, la primera de las causas queda, en cierta manera, compensada por el abarrilamiento de la matriz de compactación debido al mayor desgaste en la zona central de la misma. Para limitarlo y facilitar la compactación, a los polvos se les añaden lubricantes. En general, se trata de estearatos de bajo punto de fusión (2), que se eliminan con un calentamiento previo a la sinterización a temperaturas relativamente bajas $\left(\cong 400-600^{\circ} \mathrm{C}\right)$.

De acuerdo con lo expuesto anteriormente, se deduce que no todas las formas de piezas son realizables mediante el sistema de compactación uniaxial. Estas piezas deben cumplir fundamentalmente dos requisitos: uno, que no sean muy esbeltas para evitar gradientes de densidad y, además, que tengan una forma tal que permita su fácil extracción de la matriz. Las más habituales son piezas de revolución o cuadradas de formas complejas, con agujeros pasantes y de una relación sección/altura adecuada.

Los procesos de sinterización deben garantizar que las piezas obtenidas tengan densidad total, es decir, estén libres de poros que actúen como centros de tensiones localizadas y, por tanto, generadoras de fallos prematuros (3). Los compactos, en el caso de polvos atomizados en agua, pueden sinterizarse bajo distintas atmósferas o bien en vacío.

En un acero convencional, entendiéndose por tal un acero de forja, palanquilla o barra, los tratamientos térmomecánicos por los que deberá atravesar el material hasta llegar a la pieza final, pueden enumerarse de la siguiente forma:

- Solubilización de segregaciones o heterogeneidades químicas.

- Deformación en caliente, para disolver y romper los carburos presentes de gran tamaño.

- Recocido, para la eliminación de tensiones residuales y facilitar las operaciones de mecanizado y acabado final de la pieza.

- Normalizado, para el afino del tamaño de grano.

- Temple de austenización, para la total retención de la austenita y disolución de cierto porcentaje de carburos primarios.

- Revenidos múltiples, para la transformación a martensita precipitada de carburos secundarios.

Para piezas obtenidas por PM, los dos primeros puntos no son aplicables, si bien, cualquiera que sea su proceso de fabricación, las piezas deben someterse a diferentes tratamientos térmicos, fundamentales para lograr propieđ̛ades mecánicas adecuadas. Como consecuencia de ello se producirán cambios dimensionales, tales como:

- Cambios volumétricos por transformaciones de fases.

- En el caso de acero convencionales, variaciones en los cambios volumétricos a causa de segregaciones.

- Distorsiones debidas a tensiones residuales generadas por mecanizado, compactado o calentamientos inadecuados.

Se propone la fabricación de estas matrices de estampación y piezas similares para otros usos por procesos de metalurgia de polvos de aceros rápidos sinterizados en una atmósfera industrial rica en nitrógeno (4-6). Esto proporcionará una serie de ventajas, como son: 
- Menores costos de material.

- Ahorro derivado de la mano de obra y de máquinas.

- Mejora de las propiedades microestructurales y, por lo tanto, de las propiedades mecánicas.

- Respuesta más eficaz ante los tratamientos térmicos y sus consecuentes cambios dimensionales.

- Obtención de piezas casi listas para su uso inmediato.

El uso de distintos tipos de aceros rápidos (grados T modificado y grados $\mathrm{M}$ )(5) permite contrastar el comportamiento de cada uno de ellos en lo que respecta a contracciones durante la sinterización, cambios volumétricos en los tratamientos térmicos, microestructuras resultantes, propiedades mecánicas, etc.

La utilización de distintas atmósferas de sinterizado hace posible una variación de microconstituyentes que llevará a una mejora de algunas propiedades mecánicas. Tal es el caso de la atmósfera industrial con base de $\mathrm{N}_{2}\left(90 \% \mathrm{~N}_{2}-9 \% \mathrm{H}_{2}-1 \%\right.$ $\mathrm{CH}_{4}$ ) debido a la cual los carburos ricos en vanadio, comúnmente denominados $\mathrm{MC}$, sufren una transformación a carbonitruros (MX), aumentando, entre otras cosas, la resistencia al desgaste.

Puesto que se someten a distintos tratamientos de calentamiento y enfriamiento hay que tener en cuenta que se producen distorsiones y cambios volumétricos, tanto durante el sinterizado como durante los tratamientos térmicos, sobre todo en la transformación austenita $\rightarrow$ martensita a lo largo de los revenidos, la cual implica un incremento de volumen debido a la menor densidad de la martensita, si bien hay que destacar que las variaciones de volumen netas, debidas a los tratamientos térmicos, son despreciables.

\section{PROCEDIMIENTO EXPERIMENTAL}

La composición de los aceros utilizados, suministrados por la empresa Powdrex, Ltd., se muestra en la tabla I, y en el caso del acero T42, de alto contenido de vanadio, se solicitó una colada especial para este centro. A los polvos de acero PX30, M2 y M3/2, se les adicionó un 0,2\% de C elemental.

En primer lugar, los aceros se sometieron a un proceso de compactación para dotarlos de una forma geométrica inicial, que tras la sinterización tomó las dimensiones finales de la pieza. Para la compactación se utilizaron matrices similares a la que se muestra en esquema en la figura 1. En un primer paso, se tapa el orificio inferior con el punzón 1 y se llena con la cantidad adecuada de polvo a través de una tolva, eliminándose el sobrante con un rasero. Posteriormente, se coloca el punzón 2 y, manteniendo fijo el punzón interior hexagonal, se compacta sucesivamente con los punzones 1 y 2 mediante compresión uniaxial en una prensa de doble efecto. La presión ejercida sobre el compacto es de $650 \mathrm{MPa}$.

Como ejemplos concretos, se diseñaron dos matrices, una para estampación de cabezas de tornillos y la otra para la fabricación de terrajas. En la matriz de estampación para cabezas de tornillos, el punzón es hexagonal con forma de tronco de pirámide en su parte central, con la sección menor hacia el punzón 1 (Fig. 2).

Para la compactación de la base de terraja, se utilizó un punzón como el que se muestra en la figura 3. Dicho punzón tiene, en este caso, forma más compleja, aunque sin conicidad. Una vez

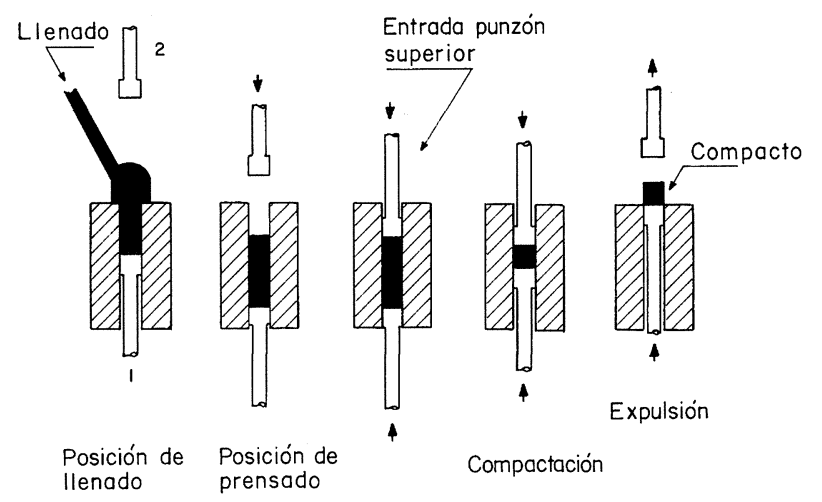

FIG. 1.- Diagrama esquemático del proceso de compactación.

FIG. 1.-Compaction process.

TABLA I.- Composición química de los polvos, \% en masa

TABLE I.-Chemical analysis of the powders. Weight percentage

\begin{tabular}{|l|ccccccccccc|}
\hline & $\mathrm{C}$ & $\mathrm{Cr}$ & $\mathrm{Co}$ & $\mathrm{Mo}$ & $\mathrm{Mn}$ & $\mathrm{Ni}$ & $\mathrm{O}, \mathrm{ppm}$ & $\mathrm{S}$ & $\mathrm{Si}$ & $\mathrm{V}$ & $\mathrm{W}$ \\
\hline $\mathrm{P} \times 30$ & 1,33 & 4,28 & 8,70 & 5,14 & 0,23 & 0,29 & 488 & 0,015 & 0,31 & 3,32 & 6,63 \\
M3/2 & 0,98 & 4,02 & 0,37 & 6,80 & 0,18 & 0,32 & 468 & 0,018 & 0,18 & 2,94 & 6,00 \\
M2 & 0,91 & 4,05 & 0,44 & 5,07 & 0,20 & 0,26 & 525 & 0,013 & 0,27 & 1,86 & 6,28 \\
T42HV & 2,76 & 4,00 & 9,0 & 3,2 & - & - & 100 & 0,016 & - & 7,2 & 9,2 \\
\hline
\end{tabular}



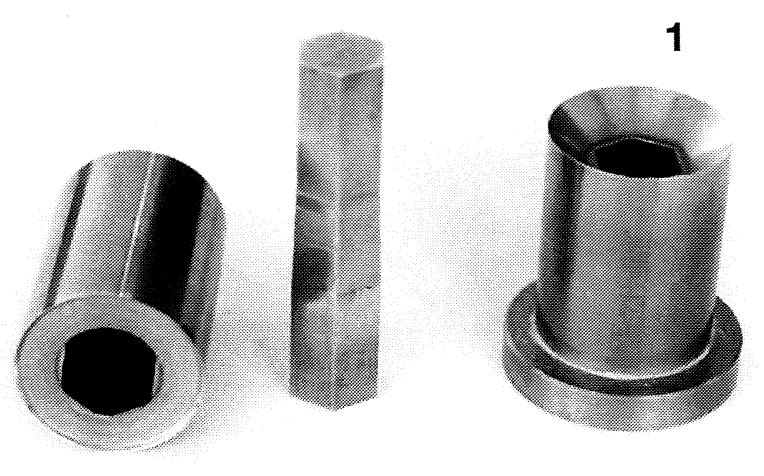

FIG. 2.- Matriz de compactación para la fabricación de matrices de estampación de cabezas de tornillos.

FIG. 2.- Rigid die to the compaction of stamping dies.

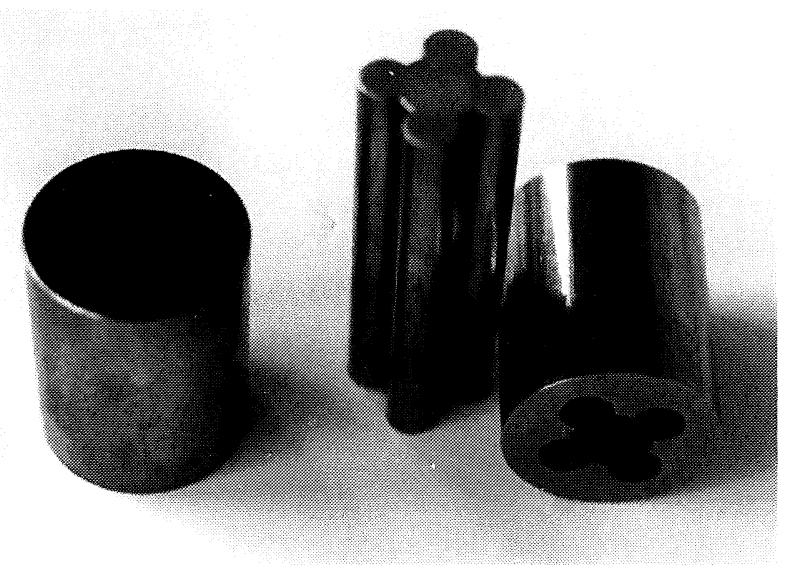

FIG. 3.- Matriz de compactación para la fabricación de terrajas.

FIG. 3.- Rigid die to the compaction of tap dies.

sinterizada la pieza, sólo resta el mecanizado de la rosca interior como paso previo a los tratamientos térmicos.

La sinterización se llevó a cabo en atmósfera industrial de $90 \% \mathrm{~N}_{2}-9 \% \mathrm{H}_{2}-1 \% \mathrm{CH}_{4}$. La presencia de hidrógeno hace a la mezcla reductora, y el metano proporciona un potencial de carbono que evita la descarburación del acero en el proceso. Durante la sinterización, se produce, por otra parte, una incorporación de nitrógeno al acero. Parte de este nitrógeno se encuentra presente en los carbonitruros MX, mientras que el restante entra en solución en la austenita.

Como resultado de la eliminación de la porosidad existente, durante el proceso de sinterizado se producen en la pieza contracciones en la pieza que hacen variar las dimensiones desde el compacto en verde hasta la pieza sinterizada. Por ello, se realizó un estudio detallado de las contracciones tras el sinterizado.
Después de la sinterización se realizaron los distintos tratamientos térmicos que a continuación se detallan:

- Recocido de esferoidización $\left(\operatorname{Tr}=800{ }^{\circ} \mathrm{C}\right)$, sólo en el caso de la terraja, con mantenimiento a esa temperatura durante $2 \mathrm{~h}$ y enfriamiento lento dentro del horno hasta temperatura ambiente. De esta forma, la pieza queda lista para el mecanizado de su rosca interior a las dimensiones adecuadas.

- Temple de austenización y disolución de carburos primarios, con enfriamiento intermedio en aceite desde la temperatura de austenización hasta 700 ${ }^{\circ} \mathrm{C}$, aproximadamente, y posterior enfriamiento en nitrógeno líquido. Este paso intermedio evita la formación de grietas y deformaciones debido al menor gradiente de temperaturas desde la de austenización hasta $700{ }^{\circ} \mathrm{C}$. El posterior enfriamiento en nitrógeno líquido, además de retener la austenita, evita la formación de carburos indeseados, tal como el $(\mathrm{Fe}, \mathrm{Cr})_{7} \mathrm{C}_{3}$, que nuclea y crece en las fronteras de grano fragilizando el material y restando cromo a la matriz.

- Revenidos múltiples, de $1 \mathrm{~h}$ cada uno. Debido al elevado contenido de carbono, la temperatura de transformación final de la martensita $\left(M_{\mathrm{f}}\right)$ se desplaza a valores muy por debajo de la temperatura ambiente. Por ello, se recurrió nuevamente a enfriamientos en nitrógeno líquido con el fin de transformar la mayor cantidad de austenita posible. Puesto que el gradiente de temperaturas es elevado, se evita la rápida estabilización de la austenita, ya que es bien sabido que cuanto mayor sea la severidad del medio de enfriamiento, menor es el tiempo en el que la austenita permanece en un rango de temperaturas adecuado para su pronta estabilización. Las temperaturas de los tratamientos térmicos para cada material se muestran en la tabla II.

TABla II.- Temperaturas óptimas de sinterizado, austenizado y revenido de los distintos aceros

TABLE II.- Optimum sintering temperatures, austenitizing and tempering temperatures for the different steels

\begin{tabular}{|c|c|c|c|c|}
\hline Material & $\begin{array}{c}\text { Temp. de } \\
\text { sinterizado, } \\
{ }^{\circ} \mathrm{C}\end{array}$ & $\begin{array}{c}\text { Temp. de } \\
\text { austenizado, } \\
{ }^{\circ} \mathrm{C}\end{array}$ & $\begin{array}{c}\text { Medio de } \\
\text { temple }\end{array}$ & $\begin{array}{c}\text { Temp. de } \\
\text { revenido, } \\
{ }^{\circ} \mathrm{C}\end{array}$ \\
\hline $\mathrm{M} 3 / 2$ & 1.240 & $1.230,1.100$ & $\begin{array}{c}\text { agua, } \\
\text { nitróg., } \\
\text { aceite }\end{array}$ & 460, \\
\hline $\mathrm{M} 2$ & 1.250 & $1.240,1.100$ & $\begin{array}{c}\text { agua, } \\
\text { nitróg., } \\
\text { aceite }\end{array}$ & $\begin{array}{c}500,540, \\
580\end{array}$ \\
\hline T42HV & 1.125 & $1.125,1.035$ & $\begin{array}{c}\text { agua, } \\
\text { nitróg., } \\
\text { aceite }\end{array}$ & \multirow{2}{*}{620} \\
\hline
\end{tabular}


Utilizando técnicas de desbaste con papeles de lija y pulido final con diamante, se prepararon secciones transversales de cada material destinadas a su observación metalográfica.

Por medio de un difractómetro de rayos $\mathrm{X}$ se midieron los picos de difracción de austenita retenida después del temple, y se siguió la transformación a martensita durante los revenidos múltiples; con ayuda de un programa desarrollado en el C.E.I.T., se calcularon los porcentajes finales de cada fase después de los tratamientos térmicos.

En estado de pulido, con un microscopio electrónico de barrido, se evaluó la presencia de porosidad residual, microestructura y distribución y tamaño de los diferentes carburos presentes.

\section{RESULTADOS}

Los valores de los cambios dimensionales producidos por la sinterización de los compactos son los que se muestran en la tabla III. Los valores son distintos para cada material, pero permanecen dentro de un mismo orden de magnitud. Es de destacar que los porcentajes de contracción de las superficies planas son diferentes a los de las superficies curvas. En la figura 4 se muestran las piezas en estado de sinterizado.

Observaciones a través del microscopio electrónico de barrido muestran carburos del tipo M6C (ricos en volframio y/o molibdeno), carburos de vanadio, MC (sólo en el caso del T42HV), y carbonitruros denominados MX (ricos en vanadio), cuya distribución es homogénea y se distinguen de los MC por su mayor contraste. La porosidad es despreciable, del tipo cerrado circular, indicando los radios de curvatura negativos que se trata de porosi-

TABla III.- Contracciones en las matrices de estampación

TABLE III.-Contractions in the stamping dies

\begin{tabular}{|c|c|c|c|}
\hline Material & $\begin{array}{c}\text { Diámetro tras } \\
\text { compactación, } \\
\mathrm{mm}\end{array}$ & $\begin{array}{c}\text { Contracción } \\
\text { media, \% }\end{array}$ & $\begin{array}{l}\text { Contracción } \\
\text { volumen, \% }\end{array}$ \\
\hline M2 & $\begin{array}{l}18,4 \\
14,65 \text { e.c.* } \\
16 \text { e.c.* } \\
31,5 \\
33,4\end{array}$ & $\begin{array}{c}13,04 \\
11,26 \\
10,0 \\
9,469 \\
10,079\end{array}$ & 27,4 \\
\hline $\mathrm{M} 3 / 2$ & $\begin{array}{l}18,45 \\
14,5 \\
16,0 \\
31,5 \\
33,15\end{array}$ & $\begin{array}{r}10,195 \\
7,965 \\
8,430 \\
8,570 \\
7,630\end{array}$ & 25 \\
\hline
\end{tabular}

e.c. $=$ Entre caras del agujero pasante hexagonal.

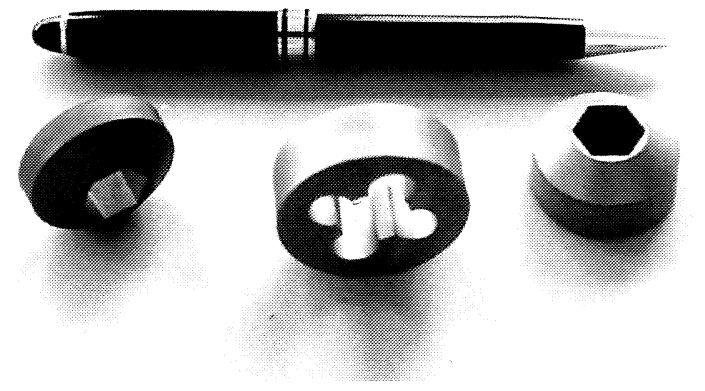

FIG. 4.- Piezas obtenidas a partir de las matrices diseñadas tras la sinterización en atmósfera industrial.

\section{FIG. 4.- Different pieces sintered under industrial atmosphere.}

dad residual. No se encontraron indicios de porosidad interconectada, lo que indicaría que la pieza no se encuentra en el punto o temperatura óptima de sinterizado (TOD). En la figura 5 se pueden ver las distintas microestructuras para cada uno de los aceros utilizados, todas ellas en la TOD. En las muestras sinterizadas, se observan carburos distribuidos de forma homogénea, tanto en los bordes de grano como en la matriz austenítica.

Si el enfriamiento tras el sinterizado es lo suficientemente lento (6), es posible que tenga lugar la formación del carburo $(\mathrm{Fe}, \mathrm{Cr})_{7} \mathrm{C}_{3}$, ya que se permite la difusión de carbono necesario para la nucleación y crecimiento del carburo. Tras el temple de la austenización, no existe ningún indicio de la presencia del carburo ( $\mathrm{Fe}, \mathrm{Cr})_{7} \mathrm{C}_{3}$ (Fig. 5).

En las micrografías de la figura 6 , se muestran microestructuras en estado de austenizado, templado y revenido. Un ataque con Nital $3 \%$ revela los bordes de grano y delinea los contornos de los carburos. Una vez austenizadas y templadas las probetas, la microestructura es esencialmente la misma que en estado de sinterizado, solo que con tamaños de grano ligeramente mayores. En el caso del acero T42HV, se registran valores de hasta un $80 \%$ de austenita retenida.

En estado de triple revenido, en todos los casos, la microestructura está formada por una matriz martensítica altamente revenida, con una fina precipitación secundaria de carburos distribuidos indistintamente en los bordes de grano y en la matriz. Sin embargo, hay que destacar que el elevado contenido de carbono del acero T42HV hace que la martensita resulte en forma de placas, gran parte de las cuales se hallan presentes incluso después de los múltiples revenidos.

Tras los revenidos, la transformación de la austenita $\rightarrow$ martensita es casi total. En la figura 7 , puede verse la evolución de la dureza con la 

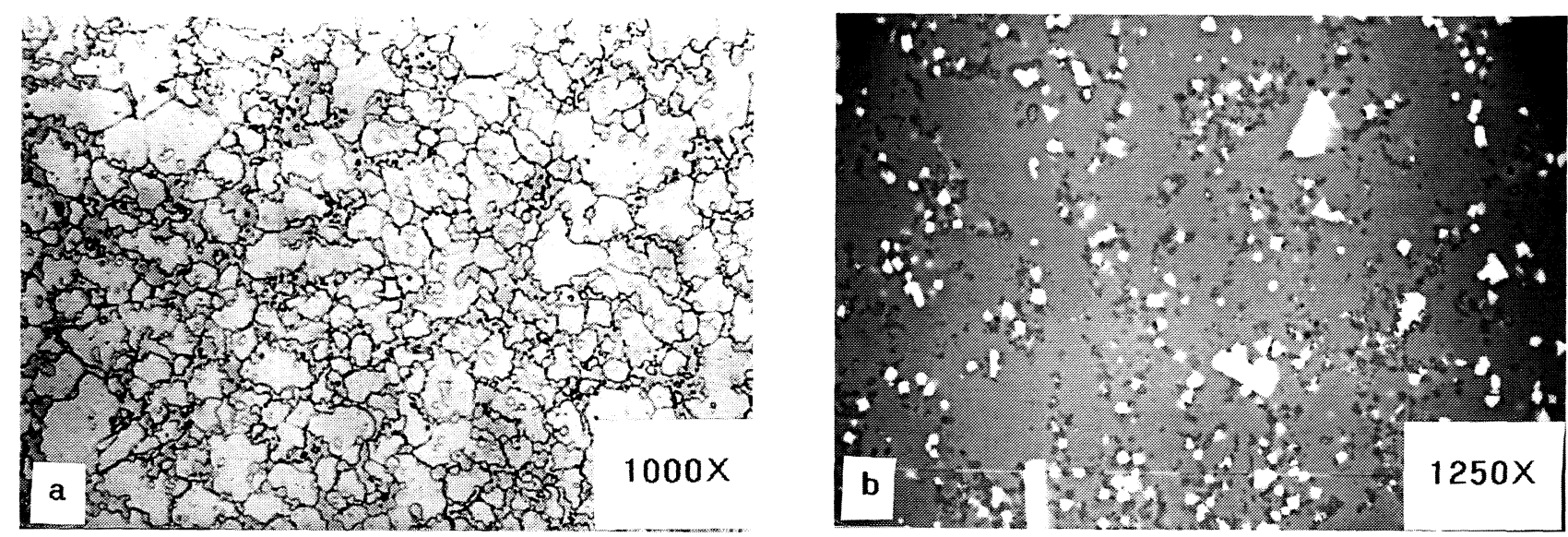

Fig. 5.- Microestructura del acero T42HV sinterizado a la TDO, $1.135^{\circ} \mathrm{C}$.

a) Microscopio óptico. Ataque Nital $3 \% . \times 1.000$.

b) Imagen con SEM. $\times 1.250$.

FIG. 5.- Microstructure of T42HV steel. Optimum sintering temperature: $1.135{ }^{\circ} \mathrm{C}$.

a) Optical. Nital $3 \% .1 .000 \times$.

b) SEM. $1.250 \times$.
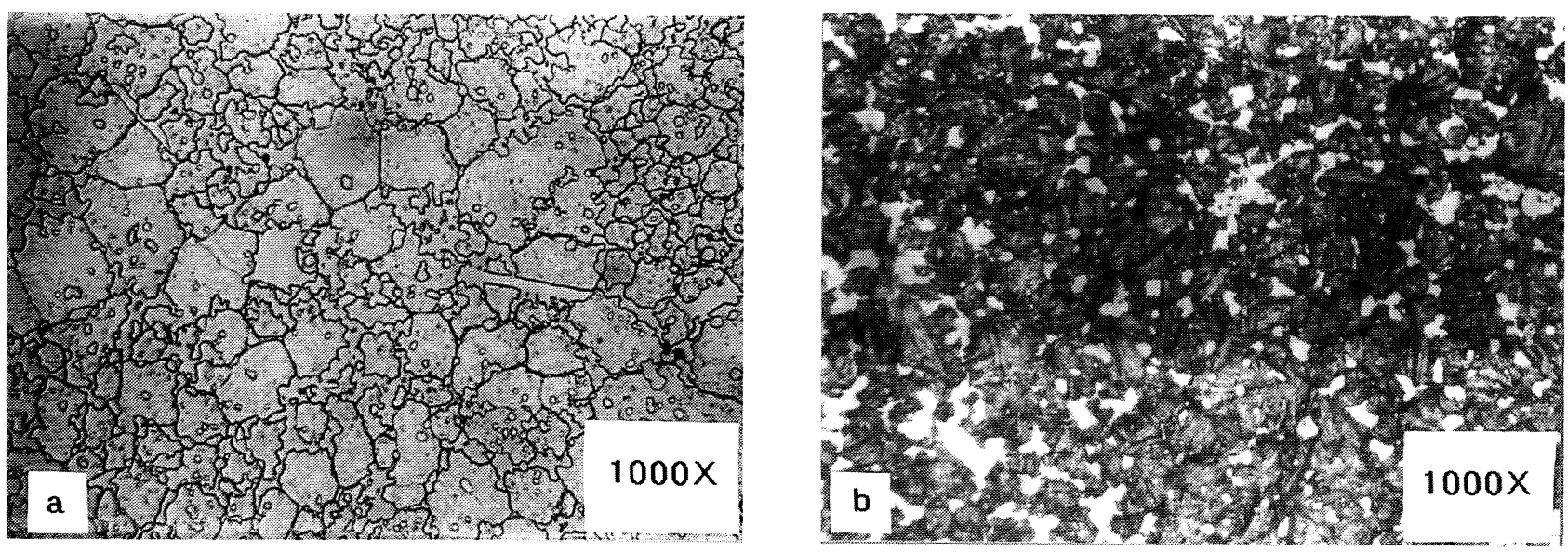

FIG. 6.- Microestructura después de los tratamientos térmicos. Microscopio óptico. Ataque Nital $3 \% . \times 1.000$.

a) Austenizado a $1.045^{\circ} \mathrm{C}$ y templado en aceite.

b) Triple revenido en aire a $540{ }^{\circ} \mathrm{C}$.

FIG. 6.-Microstructure after heat treatment. Optical microscopy. Nital $3 \% .1 .000 \times$.

a) Austenitized at $1.045^{\circ} \mathrm{C}$ and oil quenched.

b) Triple aire tempered from $540{ }^{\circ} \mathrm{C}$.

temperatura de revenido para cada uno de los aceros estudiados. A medida que la temperatura aumenta, también lo hace la dureza. Una vez que se llega al punto de máxima dureza, ésta cae rápidamente. Su disminución, a partir de temperaturas mayores que las del pico de dureza, se debe, principalmente, a un sobrerrevenido de la martensita junto con un engrosamiento de los carburos secundarios.

La transformación de la austenita, sumada al endurecimiento secundario, hace posible llegar a valores de dureza de hasta $1.100 \mathrm{HV}$ (2) en el caso del acero T42HVII. Este elevado valor puede alcanzarse con austenizaciones a temperaturas muy cercanas a la de sinterizado y con una aceleración de la transformación martensítica mediante enfriamiento en nitrógeno líquido. Sin embargo, esta rápida transformación austenita $\rightarrow$ martensita va acompañada por cambios volumétricos bruscos que pueden generar grietas, tanto internas como externas, que afectan seriamente a las propiedades finales de las piezas. Por ello, en el presente trabajo se emplearon medios de enfriamiento menos severos, tales como 


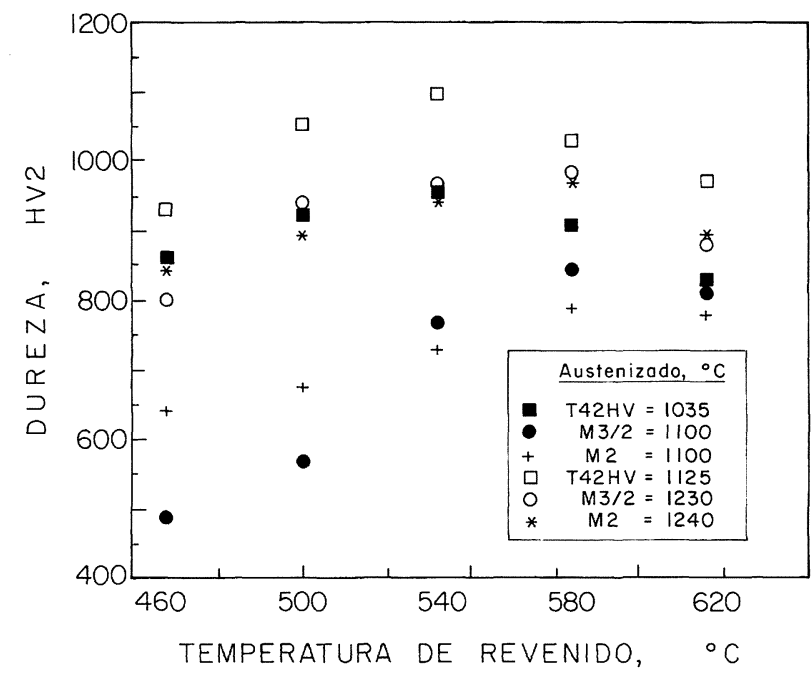

Fig. 7.- Curvas de endurecimiento de los aceros para diferentes temperaturas de austenización T42HV, M2 у M3/2.

FIG. 7.- Steels T42HV, M2 and M3/2. Hardening curves for different austenitizing temperatures.

el temple en aceite y los enfriamientos intermedios de los revenidos en aire, sin que ello signifique un freno a la transformación martensítica.

Debe destacarse que, tras los tratamientos térmicos, las distorsiones resultan inapreciables.

\section{CONCLUSIONES}

Con la metalurgia de polvos se obtienen piezas totalmente densas, de formas geométricas variadas, e incluso es posible producir conicidad en los agujeros interiores pasantes con cierta precisión.

Debido a que los cambios de volumen de las piezas tras la sinterización dependen de sus diámetros y forma geométrica, la matriz de compactación no es una reproducción a mayor escala de la pieza final.

La microestructura resultante, tras los tratamientos térmicos, es homogénea en todas las secciones de las piezas.

Los revenidos múltiples en nitrógeno líquido permiten acercarse a la $M_{\mathrm{f}} \mathrm{y}$, por lo tanto, la transformación es casi total; además, el tiempo de permanencia de la austenita a temperaturas que permiten su rápida estabilización es menor.

Las contracciones son diferentes en superficies lineales que en superficies circulares y tanto en el sinterizado como en los tratamientos térmicos son diferentes para cada material, aunque del mismo orden de magnitud.

El proceso de fabricación de estas piezas mediante metalurgia de polvos resulta ventajoso desde diversos puntos de vista, tales como homogeneidad microestructural $\mathrm{y}$, por lo tanto, de propiedades mecánicas, por el ahorro de mano de obra, de maquinaria especial y de material, y por la posibilidad de obtener formas variadas casi listas para su uso.

\section{REFERENCIAS}

(1) LENEL, F.V. Powder Metallurgy and Applications. MPIF. Princeton, N.J. 1980.

(2) German, R.M. Powder Metallurgy Science. MPIF. Princeton, N.J. 1994.

(3) Amador, J., Talacchia, S., Linaza, A., Rodriguez, J.M. y Urcola, J. Anal. Mecánica de la Fractura. Vol. 11. San Sebastián. Mar., 1994: 156-163.

(4) Palma, R. Tesis Doctoral. ESII, Univ. de Navarra. 1990.

(5) Jauregi, S., Fernandez, F., Palma, R., Martinez, V. y Urcola, J. Metall. Trans., 23A (Feb.), 1992: 389-399.

(6) Urrutibeaskoa, I., Jauregi, S., Fernandez, F., Talacchia, S., Palma, R., Martinez, V. y Urcola, J. Int. J. Powder Metall., 29 (4), 1993: 367-377. 